\title{
Les conséquences politiques de la traduction néomanagériale de la compensation : l'impensé systémique
}

Political consequences of biodiversity offsetting implementation : a neomanagerial overlooked tool

\section{Rémy Petitimbert et Clémence Guimont}

\section{OpenEdition \\ Journals}

Édition électronique

URL : http://journals.openedition.org/developpementdurable/17493

DOI : 10.4000/developpementdurable.17493

ISSN : $1772-9971$

Éditeur

Association DD\&T

\section{Référence électronique}

Rémy Petitimbert et Clémence Guimont, «Les conséquences politiques de la traduction

néomanagériale de la compensation : l'impensé systémique », Développement durable et territoires [En ligne], Vol. $11, n^{\circ} 2$ I Juillet 2020, mis en ligne le 24 juillet 2020, consulté le 26 juillet 2020. URL : http:// journals.openedition.org/developpementdurable/17493; DOI : https://doi.org/10.4000/ developpementdurable.17493

Ce document a été généré automatiquement le 26 juillet 2020.

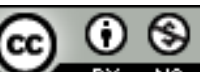

Développement Durable et Territoires est mis à disposition selon les termes de la licence Creative Commons Attribution - Pas d'Utilisation Commerciale 4.0 International. 


\title{
Les conséquences politiques de la traduction néomanagériale de la compensation : l'impensé systémique
}

\author{
Political consequences of biodiversity offsetting implementation : a neo- \\ managerial overlooked tool
}

Rémy Petitimbert et Clémence Guimont

Ce texte fut publié, la première fois, le 30 novembre 2018 : Les conséquences politiques de la traduction néomanagériale de la compensation : l'impensé systémique (vol. $9, \mathrm{n}^{\circ} 3$ )

«Baromètre : instrument ingénieux qui indique la sorte de temps que nous sommes en train de subir »

Ambrose Bierce, Le dictionnaire du diable, 1906

« Achetez de la terre, on n'en fabrique plus »

Mark Twain

1 La compensation écologique «se fixe pour objectif de réconcilier les enjeux de développement économique et de conservation de la biodiversité " (Levrel et al., 2015 : 280). Depuis la loi relative à la protection de la nature en 1976, elle constitue la troisième phase de la séquence « Éviter, Réduire, Compenser » (ERC), destinée à endiguer la fragilisation de la biodiversité dans le cadre des projets d'aménagement. Son cadre d'application est toutefois resté relativement imprécis et la compensation faisait l'objet de mise en œuvre différenciée selon les territoires et les acteurs concernés. La loi pour la reconquête de la biodiversité de 2016 reconnaît dans ses motifs l'urgence de la crise de biodiversité et la nécessité de l'endiguer. Elle prolonge l'esprit de 1976 en précisant les conditions de mise en œuvre de la séquence ERC. La réglementation rend alors obligatoires (Bigard et al., 2017) des éléments de la séquence ERC, notamment l'équivalence écologique (les gains écologiques doivent être au moins égaux aux pertes) et le principe d'absence de perte nette ${ }^{1}$. À travers le principe de " pas de perte nette ", 
la loi de 2016 impose une compensation «équivalente ». L'absence de perte nette impose que "Les mesures de compensation des atteintes à la biodiversité visent un objectif d'absence de perte nette, voire de gain de biodiversité. Elles doivent se traduire par une obligation de résultats et être effectives pendant toute la durée des atteintes » (d'après l'article L. 163-1.-I). L'écologue Baptiste Regnery explique que «le principe d'absence de perte nette permet de faire évoluer les pratiques de compensation d'un objectif de moyen à un objectif (voire une obligation) de résultat écologique » (2017:82).

2 Les mesures compensatoires, qui sont la mise en œuvre pratique de la compensation, sont conduites «en dernier recours [...] pour apporter une contrepartie positive si des impacts négatifs persistent, visant à conserver globalement la qualité environnementale des milieux»" (ministère de l'Environnement, de l'Énergie et de la Mer, 2017: 1). Elles consistent généralement en l'identification des impacts générés par un projet de développement (économique, infrastructurel, etc.) à des fins de réhabilitation des dommages ainsi générés. Elles reposent sur le principe du "pas de perte nette» et sont pour cela fondées sur la quantification des dommages résiduels des activités humaines dès lors qu'elles sont soumises à étude d'impact.

3 Depuis les années 1970, les politiques publiques connaissent de profondes transformations tant dans leur finalité que dans leur mise en œuvre (Hood, 1995). La

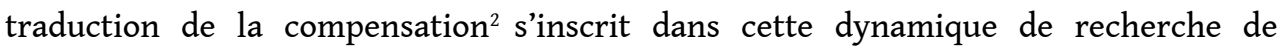
performance de l'action publique liée à la montée en puissance du New Public Management (NPM). Ces transformations ont pour effet d'aligner les procédures de décision et d'intervention de l'action publique avec les normes de la "performance " (Jany-Catrice, 2012) et de la « culture du résultat » (Dreyfus, 2010). Visant à optimiser l'efficacité et l'efficience de l'action publique, ce mouvement de fond s'observe à travers la place accordée à l'évaluation (Lacouette-Fougère et Lascoumes, 2013), aux indicateurs (Desrosières, 2014) et à la diffusion du principe de la mise en concurrence comme modalité de conduite de projet (Bruno et Didier, 2013). L'action publique mobilise ainsi des instruments, des méthodes et la philosophie gestionnaire du NPM dans la fabrique des politiques de biodiversité. Tout en prenant en compte la crise de biodiversité ${ }^{3}$ - notamment dans la reconnaissance des dommages irréversibles liés à la disparition des espèces et des écosystèmes -, l'action publique s'appuie sur des indicateurs écologiques de suivi et d'évaluation des politiques publiques (Bouleau et Deuffic, 2016) et promeut des méthodes d'ingénierie et de gestion de la biodiversité (Granjou, 2013).

4 Nous proposons d'analyser les conséquences de la mise en œuvre de cette culture du résultat sur la gestion politique de la crise de biodiversité. Pour cela, nous étudions la traduction de la compensation en mesures compensatoires par les cabinets de conseils spécialisés en ingénierie écologique. Les consultants de la compensation sont en effet au cœur de la détermination des mesures compensatoires ${ }^{4}$ (Lucas, 2015 : 37). Nous faisons dès lors l'hypothèse que, soumis à la contrainte commerciale, les consultants cherchent à développer des instruments en adéquation avec le référentiel du NPM ayant cours au sein de l'action publique. Ils influencent en conséquence l'action publique tant dans sa perception de la compensation que dans les modalités d'action à mettre en œuvre pour répondre à ce problème public. Ces traductions sont identifiées comme des instruments d'action publique (Lascoumes et Le Galès, 2004), en ce qu'elles disposent des trois propriétés identifiées par A. Hatchuel et P. Weil (cité in Lascoumes et Simard, 2011:18): "un substrat technique", "une philosophie gestionnaire" et une 
"représentation simplifiée de l'organisation"». Envisager les mesures compensatoires comme des instruments d'action publique permet d'analyser la phase de construction du problème public « compensation " par le prisme de l'instrument qui contribue à son cadrage (Gilbert et Henry, 2012). Nous entendons par là que les mesures compensatoires "entraine(nt) [...] avec elle(s) une certaine distribution des rôles entre acteurs, une hiérarchisation de ceux-ci ainsi qu'un certain type de distribution de responsabilités » (Gilbert et al., 2009 : 16-17). En cela, les mesures compensatoires - par le biais des consultants qui les élaborent - jouent un rôle politique important.

5 Il s'agit dans cet article de mettre en exergue le décalage entre les origines politiques d'un instrument - liées au contexte politique contraint par la crise de biodiversité - et les conséquences de sa mise en œuvre. Ce décalage est étudié au travers d'une analyse complémentaire entre sociologie des instruments, précédemment décrite et éthiques environnementales. Celles-ci, issues d'un courant anglo-saxon développé depuis les années 1970, interrogent pour leur part les valeurs éthiques guidant les rapports que nos sociétés entretiennent avec la biodiversité (Hess, 2013). Le dialogue entre sociologie des instruments et éthiques environnementales offre des clés de compréhension sur le cadrage politique de la crise de biodiversité qui découle de l'élaboration des mesures compensatoires.

6 La démonstration s'appuie sur un travail d'enquête de terrain, réalisée entre janvier 2015 et juin 2017, associant 24 entretiens semi-directifs auprès d'acteurs de la compensation $^{5}$. Tous ces acteurs ont participé plus ou moins directement aux différentes étapes de la rédaction de la loi pour la reconquête de la biodiversité, de la nature et des paysages de 2016 ou à la Commission d'enquête sur la réalité des mesures de compensation des atteintes à la biodiversité engagées sur des grands projets d'infrastructures en 2016-2017. Ils suivent ou accompagnent les traductions opérationnelles de la compensation soit au sein de l'État (services instructeurs, Commissariat général au développement durable, service ministériel, élu national) soit en tant que prestataires de services (cabinets de conseil, experts). Ces entretiens ont été complétés d'observations menées lors de trois événements ${ }^{6}$ dédiés à la compensation ou à la séquence "Éviter, Réduire, Compenser ${ }^{7}$ ». Les entretiens et observations ont ensuite été complétés d'un travail d'analyse d'archives telles que les comptes rendus des réunions de la commission d'enquête sénatoriale et son rapport d'enquête (Dantec, 2017).

7 Si elles sont disparates dans leurs contenus, les mesures compensatoires en cours de traduction par les cabinets sont établies à partir du principe de quantification de la biodiversité et reposent sur le principe de substituabilité entre capital naturel et capital technique. Nous démontrerons qu'elles répondent en ce sens aux préceptes d'efficience et d'efficacité du NPM (partie 1). La traduction de la compensation dans un contexte de NPM a des conséquences sur l'action politique et les acteurs qui l'animent. Elle tend à valoriser l'expression économique de la restauration de la biodiversité par rapport à sa valeur systémique, dans un contexte d'érosion et de fragilisation des milieux naturels. Les interdépendances entre milieux naturels et milieux sociaux sont alors réduites à une expression économique. Cela contribue à minimiser les irréversibilités écologiques et le rôle des humains dans la crise de biodiversité (partie 2). 


\section{La traduction néo-managériale de la compensation : quantification et substituabilité de la biodiversité}

Depuis la loi de 1976 relative à la protection de la nature, les lignes directrices nationales sur la séquence «ERC » (CGDD, 2013) précisent trois types de mesure sur les écosystèmes : leur création, leur restauration et enfin l'évolution des modalités de leur gestion. Il apparaît que la séquence n'est pas équilibrée entre ses parties, car la compensation s'impose au détriment de l'évitement et de la réduction. La loi de 2016, portant sur la reconquête de la biodiversité et des paysages, précise les frontières de leur application, mais la compensation reste privilégiée dans la mise en œuvre des mesures. En effet, malgré les tentatives de définitions via la doctrine ou les lignes directrices, l'évitement et la réduction restent difficiles à caractériser. Geneviève Gaillard, députée, rapporteure de la loi de 2016 nous confie ainsi :

«J'aurais aimé trouver des moyens de qualifier l'évitement, de qualifier la réduction. C'est quasiment pas possible. Parce que... éviter c'est éviter. Vous... éviter... comment vous voulez qualifier et définir un évitement? C'est un évitement ! La réduction, ben c'est pareil ! Il y a des études qui sont faites en amont du projet, réduire c'est réduire! Donc... on ne peut pas se permettre... mettre des pourcentages, dire "on réduit de $5 \%$ ou $10 \%$ " ça rime à rien !8. »

Elle, ajoute, fataliste :

«On a fait le constat que la séquence ERC de la loi de 1976 n'avait pas donné beaucoup de résultats. Donc ce que l'on a voulu faire c'est insister dessus et donner une méthode et vraiment des grandes lignes de compensation. Parce que sinon comment on compense ?" "

10 Et comme le précisent Charlotte Bigard et al. (2018) à propos des ambiguïtés et du déséquilibre inhérent à la constitution de la séquence ERC : «La loi biodiversité apporte quelques améliorations, notamment pour renforcer la phase d'évitement, pour suivre et évaluer les mesures mises en cuvre ainsi que pour anticiper la compensation, mais elle se concentre particulièrement sur la phase de compensation et n'apporte aucun élément réglementaire pour mieux cadrer l'interprétation faite des mesures ERC. » $(2018: 16)$.

11 Le législateur a ainsi choisi de se concentrer sur la définition des mesures compensatoires pour garantir que cet élément, pouvant devenir le seul réel opérant, puisse servir les objectifs d'absence de perte nette. Il nous semble toutefois que cela puisse aussi s'expliquer par le fait que les mesures compensatoires répondent aux canons néomanagériaux ${ }^{10}$ qui guident l'action publique. Elles entérinent en effet deux dimensions clés de cette vision, à savoir d'une part l'intensification de la quantification de la biodiversité (1.1) et d'autre part l'instauration d'un principe de substituabilité entre capital naturel et capital technique (1.2).

\subsection{Un instrument de quantification lié à un impératif de performance}

Inspirés des méthodes managériales du secteur marchand privé, les outils d'évaluation sont une modalité de management des politiques publiques (Bezes, 2012). Ils s'accompagnent de l'introduction des trois « $\mathrm{E} »$ : economy, efficiency, effectiveness (Michael Porter in Jany-Catrice, 2012). Ces trois « $\mathrm{E} »$ caractérisent la nécessité pour 
l'action publique de développer la mise en œuvre d'indicateurs de gestion budgétaire et comptable.

Selon la juriste M. Lucas (2015), le droit positif français spécifie que la compensation appartient au domaine de l'évaluation sur l'environnement, entérinant de fait une mise en œuvre de la notion fondée sur des pratiques technocratiques. Celles-ci sont fondées sur des indicateurs visant l'efficacité et l'efficience. Ainsi, si les possibilités normatives de traduction de la compensation envisagées par les cabinets sont plurielles - par l'offre ou par la demande, par les services écosystémiques, par les fonctionnalités écologiques, via des opérateurs de compensation ou directement sur le lieu impacté, etc. ${ }^{11}$,- les mesures compensatoires reposent sur des modalités de quantification tels les indicateurs ou encore les tableaux de bord d'espèces et d'habitats, visant à établir les modalités de compensation pouvant être mises en œuvre. La logique de performance du NPM décrite par Robert Salais (2010) est ici à l'œuvre : ces outils de gestion et de quantification deviennent alors des objectifs à atteindre et non plus des modalités de prise de connaissance du réel. La compensation répond aux qualificatifs gestionnaires tels qu'identifiés par Ève Chiapello et Patrick Gilbert (2013), reprenant Fayol, en ce qu'elle reprend des "principes d'administration générale": " prévoir », « organiser », « coordonner » et " contrôler » (2013: 33). Tous ces éléments sont ainsi résumés par ce consultant: "On essaie de percevoir les espaces et les espèces impactés, on établit alors le coût que ça [la compensation] va avoir pour chaque typologie préalablement construite, puis en fonction on chiffre des scénarios de réalisation. Et ça, on est capable de le faire avant la mise en ceuvre [de l'opération d'aménagement] et sur des projections d'une cinquantaine d'années avec précision ${ }^{12}$. » Le " chiffrage », pour reprendre l'expression de notre enquêté, apparaît comme un élément important pour l'établissement des mesures compensatoires. Le maître d'ouvrage, souvent l'aménageur, cherche en effet dans la majorité des cas à maximiser la restauration compte tenu de ses moyens financiers. Il aime à connaître le coût que générera la compensation sur son projet afin de pouvoir maximiser son impact en termes de mesures compensatoires sous contrainte de sa dotation financière. Charge alors aux cabinets de conseil de parvenir à établir les quantités de travail humain, de mise en œuvre technique afin de pouvoir compenser les sites dégradés soumis à étude d'impact. Ainsi, la compensation peut être appréhendée comme un instrument de calcul de ratio coûts/efficacité visant à la mesure de la qualité des politiques environnementales à partir d'une division de la biodiversité en unités quantifiables, traduite monétairement.

Or, toute opération de quantification participe à construire une représentation de l'objet observé à partir d'une réduction de la réalité autour de ce qu'il est possible techniquement et souhaitable socialement d'évaluer. Desrosières (2014) le précise clairement: "La quantification, vue comme l'ensemble des conventions socialement admises et des opérations de mesure, crée une nouvelle façon de penser, de représenter, d'exprimer le monde et d'agir sur lui» (2014: 39). Véhiculant une perception de la biodiversité séquencée en unités chiffrables et chiffrées, les traductions de la compensation contribuent à la représentation d'une biodiversité pouvant être gérée tant dans son fonctionnement (cycles naturels, fonctionnalités écologiques) qu'en termes de capital naturel à maintenir (Petitimbert, 2016). Dès lors qu'une opération d'aménagement soumise à l'étude d'impact sera mise en œuvre, une évaluation de l'état de référence sera menée à partir de travaux de quantification des espèces faune/flore ou encore des habitats (Regnery, 2017 : 129). Cette quantification peut servir à la prise de décision 
quant à la réalisation du projet (Regnery et al., 2013), et permet ainsi de mener une conciliation entre les intérêts humains et les intérêts de la biodiversité à travers le principe de substituabilité.

En effet, si la compensation en France est légalement contrainte à être réalisée « en nature ", cette restauration a un coût technique (main-d'œuvre, engins et techniques mises en œuvre...) exprimé monétairement. C'est ce que nous appelons le coût de restauration de la biodiversité, qui confère à cette dernière une valeur mobilisée lors des calculs de coûts/efficacité. Ce coût exprime monétairement la capacité humaine à compenser les destructions engendrées par une réhabilitation des milieux détruits en apportant «une description des solutions de substitution raisonnables qui ont été examinées par le maitre d'ouvrage, en fonction du projet proposé et de ses caractéristiques spécifiques, et une indication des principales raisons du choix effectué, notamment une comparaison des incidences sur l'environnement et la santé humaine " (code de l'environnement, article R122-5). La compensation telle qu'elle est traduite établit donc un rapport entre les sociétés humaines et la biodiversité, qui postule que la biodiversité est substituable par la main de l'homme et que sa valeur s'appréhende à partir d'une relation aux sociétés humaines. Les mesures compensatoires ont ainsi un coût et une valeur, exprimés par l'unité socialement construite entre toutes qu'est la monnaie (Orléan, 2011 ; Simmel, 1900). Procéder de la sorte restreint la vision de la nature à un capital qu'il serait possible de gérer et de remplacer par de la technique, par des éléments construits et établis par la main humaine via notamment un prix exprimé monétairement. Cela a un effet mécanique simple qui consiste à circonscrire la valeur de la biodiversité à son coût de restauration et à juger de l'acceptabilité de voir une partie de cette dernière s'éteindre, pourvu qu'une autre partie puisse être compensée techniquement.

\subsection{Une substituabilité centrée sur la valeur économique de la restauration de la biodiversité}

16 Comme l'expliquent Levrel et al., « la question de la compensation est intrinsèquement liée à celle de la substitution. Qui dit compensation suppose l'existence d'une possibilité de substitution de quelque chose qui a disparu par quelque chose de nouveau et qui vaut équivalence» (2015: 277). La substituabilité est ainsi le principe fondateur de la compensation sous contrainte de "pas de perte nette». Dès lors, la substituabilité et la quantification qu'elle présuppose font l'objet de controverses au sein des débats entre les acteurs de la compensation, qui s'opposent notamment sur la contribution de l'instrument à réduire la biodiversité à un capital.

17 En effet, d'après le principe de la durabilité faible, la substituabilité suppose que la nature peut être considérée comme un capital productif remplaçable par deux autres types de capitaux que sont le capital humain et le capital technique (Solow, 1993). Le capital naturel est alors conçu comme une matière productive dont la valeur est directement exprimée à travers son prix de remplacement sur le marché. Le capital naturel est envisagé comme un stock homogène, dont un minimum serait à maintenir afin de préserver la continuité des activités humaines. Il conviendrait ainsi d'évaluer le niveau de ce capital minimum nécessaire et de chercher à le maintenir via la main de l'homme $^{13}$; le principe de substituabilité pose donc une équivalence possible entre la biodiversité et les capitaux technique et humain, pourvu qu'un stock minimum de capital naturel soit préservé ${ }^{14}$. Ce cadre d'analyse permet alors d'envisager les mesures 
compensatoires comme reposant sur un mécanisme de substituabilité entre le capital naturel et le capital technique. Robert Elliott (1997) propose une analogie entre la valeur de la biodiversité et celle d'une œuvre d'art. Pour l'éthicien, la valeur d'une œuvre provient du processus artistique qui a amené à son accomplissement. Elle provient ainsi de l'époque à laquelle elle a pu être réalisée ${ }^{15}$, du temps nécessaire à sa réalisation et de son histoire. La valeur de l'œuvre provient également de son originalité créative : il doit s'agir d'une œuvre "originale ». Il en va de même pour la biodiversité. Sa valeur provient des processus qui ont contribué à la faire exister telle qu'elle est dans sa naturalité ontologique et qui la lient au passé. Ainsi, en mettant en œuvre des mesures compensatoires, l'homme agit en faussaire, il confère la valeur d'une œuvre par essence non reproductible - la biodiversité «naturelle» - à une contrefaçon. Comme le rappelle Éric Katz dans son texte au titre des plus évocateurs $L e$ grand mensonge, la restauration de la nature par les hommes, accepter le principe de la compensation c'est oublier le fait qu'" une nature "restaurée" est un artefact créé pour répondre aux intérêts des hommes et leur apporter une satisfaction" (Katz, 1992, 2007 : 351). Ces travaux questionnent alors la compensation en ce qu'elle devient une substitution et non plus une restauration. Cela interroge également la nature de la biodiversité "substituée ", comparativement à celle de la biodiversité initiale puisque, pour Éric Katz, "conférer à un paysage qui a été restauré la même valeur que celle de son original, c'est par conséquent [...] se laisser duper par les ressemblances superficielles avec la zone naturelle, de même que l'"esthète" ignare s'est laissé duper par l'aspect extérieur de la contrefaçon " (Katz, $2007: 354)$.

Le principe de substituabilité circonscrit donc la valeur de la biodiversité à l'appréhension sociale de sa restauration. La valeur de la biodiversité est en effet déterminée à partir d'un capital disponible sur un marché dédié. Cette circonscription de la valeur de la biodiversité à son acception économique, et dans une large part monétaire, peut procéder à une réduction des valeurs de la biodiversité autour des seuls éléments que l'action publique et ses partenaires privés sont en mesure de quantifier. Pourtant, les valeurs de la biodiversité sont multiples, en dehors même de leur valeur économique ${ }^{16}$. Il s'agit là d'une question centrale au sein des éthiques environnementales, qui, depuis le début des années 1970, s'intéressent à la délicate question de la «valeur » de la nature, au-delà de la reconnaissance de sa seule utilité pour le marché (Hess, 2013). L'éthicien Holmes Rolston propose ainsi une catégorisation mettant en exergue notamment la valeur systémique de la biodiversité, qui se définit par la reconnaissance des interdépendances entre tous les êtres vivants au sein des écosystèmes (Rolston, 2007).

La détermination des mesures compensatoires à partir d'une considération monétaire de la restauration de la valeur de la biodiversité n'est pas partagée par tous. On note deux positionnements différents parmi les acteurs concernés. La première, plutôt soutenue par les aménageurs, les hauts fonctionnaires ainsi que la majorité des bureaux d'études, tend largement à valoriser l'évaluation économique de la restauration de la biodiversité au détriment des autres possibilités d'appréhension de la biodiversité. La reconnaissance de l'intervention de nos sociétés sur l'ensemble des milieux naturels explique en partie pour eux la prédominance des estimations monétaires. Comme l'analyse cet économiste interrogé, "il s'agit de considérer que l'homme est devenu le jardinier du monde qui l'entoure. Par conséquent il est le plus apte à pouvoir compenser ce qu'il a lui-même créé ${ }^{17}$ ». Ainsi, pour les tenants de cette conception, non seulement l'humain est à l'origine de la valeur de la biodiversité - mécanisme d'attribution -, mais il en est 
par ailleurs le gestionnaire - mécanisme de régulation - et il doit en assumer la préservation - mécanisme d'évaluation. Pour eux, les actions humaines sont donc à l'origine de la valeur de la biodiversité car elles façonnent la nature pour lui donner son visage actuel et car la communauté humaine évalue la valeur de la biodiversité en fonction des services apportés. Comme nous le dit un enquêté :

«Mon travail c'est de faire des évaluations. Pour qu'il y ait évaluation, il faut qu'il y
ait un évaluateur... à partir de là, appliqué à l'environnement, pourquoi un arbre a
une valeur? Essentiellement parce qu'il capte du $\mathrm{CO}_{2}$ et que ça nous rend service à
nous [les sociétés humaines] [...] Le but est alors d'optimiser les services de
captation rendus lors d'opération de renaturation [via la sélection des espèces à
introduire en fonction de leur capacité d'absorption du carbone] ${ }^{18}$. "

Dans cette optique, la substituabilité est réalisable jusqu'à un stock critique: elle exprime la possibilité d'aménagements toujours possibles, car des solutions de restauration peuvent être trouvées.

21 La seconde position s'inscrit en faux face à une telle représentation de la biodiversité et met l'accent sur son fonctionnement plus que sur ses composantes. Soutenue majoritairement par les acteurs issus $\mathrm{du}$ monde associatif (naturalistes, environnementalistes et activistes notamment) et de quelques consultants indépendants, elle souhaite la reconnaissance et la prise en considération de la valeur systémique de la biodiversité proche de celle proposée par Holmes Rolston. Cette salariée travaillant pour une association de défense de l'environnement résume les termes du débat :

«Ce qui est embêtant c'est de penser qu'elle [l'évaluation économique de la restauration de la biodiversité] peut le faire, et de vouloir comme objectif lointain une rationalité économique totale qui réduirait toutes les valeurs à une seule dimension monétaire dans des domaines qui relèvent en réalité de l'éthique, qui sont parfois incommensurables ${ }^{19}$. "

Cette approche entend insister sur le fait qu'une des valeurs de la biodiversité qui devrait être intégrée dans la mise en œuvre de la compensation provient de la capacité des écosystèmes à se maintenir en vie à travers les interdépendances établies entre les êtres vivants et les milieux, à travers le temps écologique. La valeur de la biodiversité provient donc non seulement du fait qu'elle existe en tant que telle, par elle-même et pour elle-même, mais également par les interactions qui lui permettent d'exister. Cette perspective exposée par cette deuxième catégorie d'acteurs rejoint la position défendue par H. Rolston, qui précise ainsi : «Un écosystème est une matrice d'interconnexions où les composantes biotiques et abiotiques évoluent solidairement [...] La valeur systémique de la nature désigne la créativité qui opère au sein de la nature en vertu de laquelle viennent au jour la plus grande diversité et la plus grande complexité des formes de vie dans le cadre d'un système où rien n'est laissé au hasard, où tout joue un rôle, aussi discret soit-il, et où pourtant tout peut advenir ${ }^{20}$.»

En écho à ces propos, cet ingénieur travaillant à la traduction de mesures compensatoires au sein d'un cabinet de conseil précise que

«la richesse du vivant réside bien plus dans sa cohérence globale que dans les unités qui la composent, dans la capacité d'un écosystème à se maintenir et à se développer, dans le temps ${ }^{21}$.»

24 Ainsi, le principe de substituabilité, qui présuppose la capacité humaine à reproduire financièrement ${ }^{22}$ et techniquement tant des unités de biodiversité que les interdépendances favorisant sa permanence, peut amoindrir la valeur systémique de la 
biodiversité. Ainsi perçue, la substituabilité se centre en effet sur une valeur de "reconstruction", de reproduction d'un écosystème établi sur des temporalités propres à l'action publique (Guimont, 2018). Elle diminue alors la valeur systémique de la biodiversité qui s'établit à partir de la temporalité millénaire du vivant, élaborée par des interactions entre l'ensemble des êtres vivants humains et non humains sur un temps très long (Hess, 2015). Elle ne prend en compte ni les interdépendances entre le milieu détruit et les écosystèmes qui l'entouraient ni les temporalités nécessaires à la biodiversité pour se (re)constituer. L'écosystème compensé est ainsi copié d'après des perceptions humaines (Katz, 1992, 2007) sans que la valeur systémique de celui-ci ne lui soit reconnue.

Ainsi, la mise en œuvre de la compensation ne peut permettre de penser qu'une partie de la relation entre la biodiversité et les sociétés. En ce sens, elle contribue à atténuer le rôle du développement humain dans la crise de biodiversité en établissant la capacité des sociétés humaines à «restaurer la nature pour atténuer les impacts du développement » (Levrel et al., 2015).

Les tenants d'une vision favorable à la substituabilité, appartenant à la première catégorie d'acteurs présentés, sont majoritaires ou en capacité de mobiliser des capitaux (relationnels, sociaux, techniques) concourant à les rendre plus audibles au sein de l'action publique. Ils contribuent à asseoir l'importance de la substituabilité comme cadrage cognitif et normatif. Dès lors, la focalisation des pouvoirs publics sur les modalités néo-managériales de mise en œuvre de la compensation a pour effet de rendre invisibles dans les arènes publiques les irréversibilités écologiques liées à l'impact de nos activités sur les milieux naturels.

\section{Les conséquences politiques de la mise en œuvre de la compensation sur l'appréhension de la crise de biodiversité}

En cherchant à établir la modalité la plus efficace de compensation, les acteurs se focalisent sur les aspects techniques de l'instrument, masquant par là les questions politiques liées à la valorisation de la biodiversité à travers les cycles naturels sur lesquels il repose.

\subsection{De la logique néo-managériale à la réduction de la biodiversité par la diffusion de la « compensation »}

Les débats entre les acteurs de la compensation, notamment entre les pouvoirs publics et les cabinets d'études environnementales, se concentrent sur les modalités de mise en œuvre et participent alors à légitimer le recours à la quantification et à la substituabilité (Petitimbert, 2017). À titre d'exemple, dans le cadre du développement d'une infrastructure fluviale, un des plus importants cabinets de conseils français a travaillé à l'établissement d'un instrument d'évaluation de mesures compensatoires ${ }^{23}$. Nommé «méthode en miroir ", ce procédé consiste dans un premier temps à classifier des milieux impactés sur une échelle allant du site le plus riche au site le plus pauvre en biodiversité (et en espèces remarquables, sur liste rouge...), puis dans un second temps, d'après la même échelle, à classifier des sites pouvant servir de lieux d'accueil aux 
espèces identifiées. Une fois cette opération réalisée, charge à l'aménageur de mettre en place cette compensation. Ceci n'est pas sans poser de questions techniques quant à la possibilité d'obtention de ces sites, à leur configuration pour accueillir des espèces qui voient leur habitat dégradé, à la possibilité de voir lesdites espèces y migrer, ainsi qu'au potentiel de développement écologique de ces sites. En effet, les sites dégradés le sont aujourd'hui, tandis que le potentiel de développement du site de compensation est un pari sur l'avenir. La centralité dans les débats de ces questions techniques se constate particulièrement lors des temps d'échanges que représentent les colloques dédiés au sujet. Lors des trois colloques observés ont été principalement abordés les enjeux techniques qui visent à construire la méthode la plus précise du calcul des mesures compensatoires, par exemple sur les questions liées à la surface, le service rendu ou encore l'espèce. Ensuite, elles ont interrogé la bonne modalité de leur mise en œuvre (compensation par l'offre ou par la demande) et leur bonne traduction (monétaire ou non). Enfin, elles se sont penchées, dans une moindre mesure, sur les conditions de gestion des mesures compensatoires dans le temps.

Il s'agit d'une dimension sur laquelle nous voudrions insister. Elle marque la difficulté d'élaborer et de valoriser la compensation à travers une unité acceptée par les acteurs concernés.

Du côté des cabinets de conseils, au-delà de l'analyse des discours, il est intéressant de se pencher sur les supports de communication projetés lors des prises de parole et tables rondes; ces derniers reflétant la technicité à adopter pour être reconnu comme crédible lors de ces temps de discussion. L'analyse des supports visuels proposés par les différents intervenants pour nourrir leurs démonstrations reflète la concentration des débats autour des modalités techniques de la compensation. Les discours se trouvent complétés par des projections d'extraits de plans, de données chiffrées de cartographies, de tableaux et graphiques, ou encore de modélisations accentuant la technicité de la démonstration. La prédominance des questions techniques constitue un sésame tant pour donner des gages d'insertion au sein de la communauté que pour présenter la capacité de l'intervenant à établir des mesures compensatoires et ainsi à valoriser la modalité technique et l'offre commerciale proposées. Le consultant est alors face à un double enjeu : celui de la crédibilité technique de son offre et celui de la crédibilité commerciale en démontrant à l'assistance que son offre a vocation à être multipliée.

Du côté des pouvoirs publics, la commission d'enquête, dirigée par le sénateur Ronan Dantec, sur la réalité des mesures de compensation des atteintes à la biodiversité engagées sur des grands projets d'infrastructures (Dantec, 2017), participe à cette ambiguïté du positionnement. Cette commission avait pour objectif d'interroger les conditions de mise en œuvre et d'évolution des pratiques de la compensation; cela prenait en compte les mesures d'anticipation, les études préalables, les conditions de réalisation et leur suivi. Du 28 novembre 2016 au dévoilement du rapport le 11 mai 2017, cette commission d'enquête a rassemblé des sénateurs et sénatrices issus de différents mouvements politiques.

32 La commission a réalisé l'audition de toutes les positions en présence ${ }^{24}$ afin de pouvoir "déterminer les difficultés de mise en cuvre des mesures de compensation, et formuler des propositions facilitant la conduite de la séquence Éviter, Réduire, Compenser $(E R C)^{25} »$. Elle a par conséquent insisté davantage sur les enjeux techniques que sur la "philosophie» de la compensation. Ainsi, les questions techniques priment : « méthodologies à améliorer 
", " plus grande anticipation ", "renforcement des expertises publique et privée ${ }^{26}$ " (Dantec, 2017). L'objectif est clairement de promouvoir l'adoption de bonnes pratiques de mise en œuvre, d'associer les parties prenantes et de standardiser les mesures (Dantec, 2017 : 3). Le rapport nous semble donc s'inscrire clairement dans le sillage de l'ingénierie écologique, insistant sur l'ajustement des dimensions techniques afin d'optimiser l'efficacité de la compensation. On retrouve là, finalement, les objectifs préconisés par l'approche néomanagériale de l'efficacité de l'intervention publique, au détriment d'une approche de la valeur systémique de la biodiversité.

\subsection{Les impensés provoqués par la réduction de la biodiversité à des modalités néo-managériales} simplification et à l'occultation des relations existant entre milieux sociaux et milieux naturels, car elle contribue à l'occultation de la valeur systémique. La quantification et la substituabilité, au cœur des travaux, donnent l'idée que la biodiversité peut être reconstituée et séquencée. Mais cela masque les difficultés posées par la mise en œuvre des mesures compensatoires. Celles-ci ont des conséquences politiques et éthiques en ce que l'instrument, dès qu'il est mobilisé par l'action publique, participe à une réduction de la complexité des interactions entre milieux sociaux et milieux naturels, qui atténue la gravité de la crise de biodiversité.

La focale sur les conditions techniques de la «bonne » compensation fait obstacle à la reconnaissance de l'incapacité humaine à laisser à la biodiversité les conditions de sa pérennité et à la prise de conscience du poids des choix des sociétés humaines dans la crise de biodiversité telle que la valeur systémique le met en exergue. Nous l'avons vu, la substituabilité est source de controverses entre les acteurs selon que ces derniers la considèrent comme possible ou qu'ils estiment que la biodiversité ne peut être restaurée sans que cela ne résulte d'un travail de faussaire. Ces positions antagonistes détaillées précédemment présentent toutes deux des limites liées à la valeur de la biodiversité. Pour la première, il s'agirait d'évaluer à travers un regard monétaire la restauration de la biodiversité et pour la seconde, de considérer la biodiversité sous toutes ses dimensions, mais avec la difficulté de l'unité d'appréciation que cela implique. En outre, ces deux points de vue interrogent peu l'objectif des mesures compensatoires qui consistent à préserver un capital naturel permettant aux sociétés humaines de maintenir leurs activités. Ainsi, la question de la «bonne » compensation semble moins être une question technique qu'une question politique. Il s'agit alors, à travers l'interrogation des effets politiques des mesures compensatoires, de chercher à dépasser les impensés de la substituabilité en considérant la crise de la biodiversité dans son actualité (Ceballos et al., 2015). Le temps passé par les acteurs à décider et à élaborer les mesures compensatoires ne leur permet pas de mettre en œuvre des mesures d'évitement des dommages écologiques. Comme le démontre Fanny Guillet (2017), la focalisation des administrations publiques sur les modalités de mise en œuvre de la compensation, coûteuse sur le plan humain et financier, mobilise l'ensemble des acteurs publics et privés et empiète sur la mise en œuvre des autres politiques de biodiversité qui évitent en amont les irréversibilités écologiques, telles que les plans d'aménagement et la gestion foncière des espaces naturels.

Développement durable et territoires, Vol. 11, n² | Juillet 2020 
Enfin, les mesures compensatoires présupposent la capacité des sociétés humaines à fractionner la biodiversité en unités (espèces, milieux, etc.) qu'il conviendrait de restaurer en fonction des destructions/dommages générés par les projets menés. Cette acceptation du principe de séquençage de la biodiversité en unités substituables (liée aux principes de quantification et de substituabilité) tend à effacer le rôle majeur que jouent les interdépendances dans le fonctionnement de la biodiversité. En conséquence, les mesures compensatoires ne peuvent donc pas permettre d'appréhender la valeur systémique de la biodiversité qui trouve son fondement dans les interdépendances. Deux types d'interdépendances sont ainsi distinguables:1) les interdépendances des espèces et des espaces naturels et 2) les interdépendances des sociétés humaines et de leur environnement. Les débats occultent les interdépendances entre les sociétés humaines et leur environnement et les interdépendances de la biodiversité, qui doit s'adapter aux choix sociétaux qu'elle subit. Ces interdépendances ne peuvent être appréhendées uniquement sous l'angle d'un rapport coûts/efficacité des mesures compensatoires tel que proposé par l'ingénierie écologique au cours des débats (Levrel et al., 2015). Face à la nécessité de saisir ces interdépendances, Arne Næss, éthicien de l'environnement, propose la notion d'identification pour saisir ce qui nous lie au reste du vivant (Næss, 2013). L'identification signifie que nous ne parvenons à être nous-mêmes que par la reconnaissance d'une appartenance à un ensemble plus grand que la communauté humaine. Ce tout est celui qui rassemble l'ensemble des êtres composant la biodiversité et les êtres humains, envisagé à partir d'un futur commun. Ce futur est celui de l'évolution de la biodiversité, qui comprend l'ensemble des écosystèmes dont nous faisons partie. Il écrit ainsi : «En nous identifiant à de plus grands touts, nous prenons part à la création et au maintien de ce tout» (Næss, 2013: 276). L'identification invite à un élargissement des valeurs dans la fabrique de l'action publique. L'intégration de la valeur systémique semble alors être une piste à suivre afin d'éviter la réduction des relations existant avec la biodiversité aux logiques d'efficacité et de résultat.

\section{Conclusion}

La biodiversité se voit ainsi davantage incorporable dans les logiques néo-managériales. Celles-ci contribuent, par un mécanisme de mise à distance de la biodiversité qui caractérise le tropisme dualiste de nos sociétés modernes, à minimiser la responsabilité des sociétés humaines dans le développement et l'intensification de la crise de biodiversité. Ces approches néo-managériales valorisent, tant dans l'évaluation de la biodiversité que sa régulation et sa gestion, les approches techniques. Ces dernières ont certes l'avantage de pouvoir établir une quantification de la biodiversité qui s'inscrit dans la chaîne des instruments de l'action publique. Elles offrent aussi la vision d'une possible restauration de la biodiversité. Mais cette stratégie sous-estime les échelles du temps de la biodiversité. L'histoire longue de la biodiversité témoigne de ses capacités de permanence et d'adaptation dès lors qu'elle a la possibilité de s'insérer dans des temporalités longues.

Mais la crise de biodiversité vient bousculer non seulement les temporalités de l'action publique, mais aussi la gestion des conséquences de nos choix techniques. La compensation présuppose une temporalité linéaire dans la prise en compte des effets des mesures compensatoires. Pourtant, la crise de biodiversité oblige les sociétés 
humaines à davantage considérer l'approche temporelle en termes de délai : «Bientôt, trop tard» comme le rappelle, à nouveau, le cri d'alarme de 15000 scientifiques (Ripple et al., 2017). Le délai indique que le temps d'agir se réduit au fur et à mesure que les irréversibilités écologiques s'accumulent: "Nous sommes, selon Bruno Villalba, désormais inscrits dans un délai nous imposant de réaliser des choix qui seuls permettront la continuité réelle de l'existence de nos sociétés » (2016: 90).

L'urgence de la situation amène à penser que l'action devrait être menée également à partir d'une reconnaissance de la valeur systémique de la biodiversité. Celle-ci permet de saisir par un autre prisme les liens qui nous unissent au reste du vivant. Cela semble nécessaire pour adapter notre action à l'urgence des irréversibilités.

\section{BIBLIOGRAPHIE}

Barnosky A. D., Hadly E. A., Bascompte J., Berlow E. L., Brown J. H., Fortelius M., Getz W. M., Harte J., Hastings A., Marquet P. A., Martinez N. D., Mooers A., Roopnarine P., Vermeij G., Williams J. W., Gillespie R., Kitzes J., Marshall C., Matzke N., Mindell D. P., Revilla E., Smith A. B., 2012, « Approaching a state shift in Earth's biosphere », Nature, vol. 486, n 7401, p. 52-58.

Bezes P., 2012, «État, experts et savoirs néo-managériaux », Actes de la recherche en sciences sociales, vol. 193, p. 16-37.

Bigard C., Regnery B., Biasco F., Thompson J. D., 2017, « La prise en compte de la biodiversité dans les études d'impact : évolutions prometteuses mais lacunaires ", Revue Sciences Eaux \& Territoires, hors-série 39, p. 2-8.

Bigard C., Regnery B., Pioch S., Thompson J. D., 2018, « De la théorie à la pratique de la séquence Éviter-Réduire-Compenser (ERC) : éviter ou légitimer la perte de biodiversité ? ", Développement durable et territoires. Économie, géographie, politique, droit, sociologie, vol. 9, n 1, https:// journals.openedition.org/developpementdurable/12032.

Bouleau G., Deuffic P., 2016, « Qu'y a-t-il de politique dans les indicateurs écologiques ? », VertigO - la revue électronique en sciences de l'environnement, vol. 16, n², En ligne : http:// vertigo.revues.org/17581.

Bruno I., Didier E., 2013, Benchmarking : l'État sous pression statistique, Paris, Zones, 209 p.

Callicott J.-B., 2010, Éthique de la terre, Marseille, Éditions Wildproject, 315 p.

Ceballos G., Ehrlich P.R., Barnosky A. D., García A., Pringle R. M., Palmer T. M., 2015, « Accelerated modern human-induced species losses : Entering the sixth mass extinction ", Science Advances, vol. 1, n 5, p. e1400253.

Chiapello È., Gilbert P., 2013, Sociologie des outils de gestion : introduction à l'analyse sociale de l'instrumentation de gestion, Paris, La Découverte, 294 p.

Dantec R., 2017, Compensation des atteintes à la biodiversité : construire le consensus, Paris, Sénat, $226 \mathrm{p}$. 
De Groot R., Van der Perk J., Chiesura A., van Vliet A, 2003, « Importance and threat as determining factors for criticality of natural capital », Ecological Economics, n 44, p. 187-204.

Desrosières A., 2014, Prouver et gouverner : une analyse politique des statistiques publiques, Paris, La Découverte, $284 \mathrm{p}$.

Dreyfus F., 2011, «La révision générale des politiques publiques, une conception néolibérale du rôle de l'État? ", Revue française d'administration publique, vol. 136, p. 857-864.

Elliot R., 1997, Faking nature : the ethics of environmental restoration, Londres, Routledge, 177 p.

Gilbert C., Henry E., 2012, «La définition des problèmes publics : entre publicité et discrétion », Revue française de sociologie, vol. 53, $\mathrm{n}^{\circ} 1$, p. 35-59.

Gilbert C., Henry E., Bourdeaux I., 2009, « Lire l'action publique au prisme des processus de définition des problèmes ", in Gilbert C. et al., Comment se construisent les problèmes de santé publique, Paris, La Découverte, 290 p.

Granjou C., 2013, Micropolitiques de la biodiversité : experts et professionnels de la nature, Bruxelles, Peter Lang, $202 \mathrm{p}$.

Guillet F., 2017, « Résultats du projet de recherche COMPILSA », 19 octobre 2017, ministère de l'Écologie, programme ITTECOP.

Guimont C., 2018, « Gérer l'urgence de la disparition du vivant : les contradictions temporelles de l'action publique. ", Temporalités, vol. $28, \mathrm{n}^{\circ} 2$.

Hess G., 2013, Éthiques de la nature, Paris, PUF, 422 p.

Hood C., 1995, "The "New public management" in the 1980's : variations on a theme ", Accounting, organizations and society, vol. 20, n² 2/3, p. 93-109.

Jany-Catrice F., 2012, La performance totale : nouvel esprit du capitalisme?, Villeneuve-d'Ascq, Presses universitaires du Septentrion.

Katz E., 1992, 2007, « Le grand mensonge : la restauration de la nature par les Hommes », in Afeissa, H.-S. (dir.), Éthique de l'environnement. Nature, valeur, respect, Paris, Vrin, p. 347-371.

Lacouette-Fougère C.-L., Lascoumes P., 2013, « L'évaluation : un marronnier de l'action gouvernementale? », Revue française d'administration publique, vol. 4, n 148, p. 859-875.

Lascoumes P., 1994, L'éco-pouvoir : environnements et politiques, Paris, La Découverte, 1994, 317 p.

Lascoumes P., Le Galès, P. (dir.), 2004, Gouverner par les instruments, Paris, Presses de Science Po, $370 \mathrm{p}$.

Lascoumes P., Simard L., 2011, « L'action publique au prisme de ses instruments », Revue française de science politique, vol. $61, \mathrm{n}^{\circ} 1$, p. 5-22.

Levrel H., Frascaria Lacoste, N., Hay J. (dir.), 2015, Restaurer la nature pour atténuer les impacts du développement : analyse des mesures compensatoires pour la biodiversité, Versailles, Quæ, 313 p.

Lucas M., 2015, Étude juridique de la compensation écologique, Issy-les-Moulineaux, LGDJ Lextenso éditions, $629 \mathrm{p}$.

Mace G.M., Reyers B., Alkemade R., Biggs R., Chapin III F.S., Cornell S.E., Díaz S., Jennings S., Leadley P., Mumby P.J., Purvis A., Scholes R.J., Seddon A.W.R., Solan M., Steffen W., Woodward G., 2014, « Approaches to defining a planetary boundary for biodiversity », Global Environmental Change, vol. 28, p. 289-297. 
Maris V., 2010, Philosophie de la biodiversité : petite éthique pour une nature en péril, Paris, BuchetChastel, $213 \mathrm{p}$.

Naess A., 2013, Écologie, communauté et style de vie, Paris, Éd. Dehors, 395 p.

Naess A., 2017, Une écosophie pour la vie : introduction à l'écologie profonde, Paris, Seuil, 347 p.

Orléan A., 2011, L'empire de la valeur, Paris, Seuil, 386 p.

Petitimbert R., 2016, «Quantifier pour manager. L'émergence des paiements pour services environnementaux au sein des politiques publiques environnementales françaises ", Développement durable et territoires, vol. 7, $\mathrm{n}^{\circ}$ 1, https://journals.openedition.org/ developpementdurable/11203.

Petitimbert R., 2017, « La compensation comme instrument de management du vivant. Un point de vue depuis la science politique ", Revue juridique de l'environnement, vol. 43, n 4, p. 659-669.

Pioch S., 2017, Vers une nouvelle gouvernance côtière entre aménagement et environnement ? : la compensation des impacts de l'homme sur l'environnement dans les projets d'aménagements maritimes Synthèse de l'activité scientifique, Habilitation à diriger des thèses, Montpellier, France, 163 p.

Randall A., 1991, « The value of biodiversity », Ambio, vol. 20, n² 2, p. 64-68.

Regnery B., 2017, La compensation écologique : concepts et limites pour conserver la biodiversité, Paris, Publications scientifiques du Muséum, $287 \mathrm{p}$.

Regnery B., Kerbiriou C., Julliard R., Vandevelde J.-C., Le Viol I., Burylo M., Couvet D., 2013, «Sustain common species and ecosystem functions through biodiversity offsets : response to Pilgrim et al. », Conservation Letters, vol. 6, n 5, p. 385-386.

Ripple W., Wolfe C., Newsome T., Galetti M., Alamgir M., Crist E. Mahmoud I., Laurence W., 2017, « World Scientists' Warning to Humanity : A Second Notice », Bioscience.

Rolston H., 1994, "La valeur de la nature et la nature de la valeur », in Afeissa HS., Éthique de l'environnement. Nature, valeur, respect, Paris, Vrin, p. 153-186.

Salais R., 2010, « Usages et mésusages de l'argument statistique : le pilotage des politiques publiques par la performance », Revue française des affaires sociales, $\mathrm{n}^{\circ}$ 1-2, p. 129-147.

Simmel G., 1900, 2014, Philosophie de l'argent, Paris, PUF, 672 p.

Solow R., 1993, Macroeconomics annual, Cambridge, Mit Press, 377 p.

Villalba B. (dir.), 2009, Appropriations du développement durable : émergences, diffusions, traductions, Villeneuve-d'Ascq, Presses universitaires du Septentrion, 387 p.

Villalba B., 2016, « Temporalités négociées, temporalités prescrites. L'urgence, l'inertie, l'instant et le délai », in Hubert B., Mathieu N. (dir.), Interdisciplinarités entre natures et sociétés, Bruxelles, Peter Lang, p. 89-109.

\section{NOTES}

1. «Ce principe repose sur la possibilité de calculer une quantité de perte de milieu naturel, souvent désignée par "biodiversitê" par les aménageurs [...] entre un état initial (état de référencé) et un état final, après les impacts des travaux sur le site endommagé. Si l'objectif est de restaurer ailleurs, sur un site compensatoire, ce qui a été détruit là, l'aménageur responsable doit proposer une surface [...] dont les gains 
sont équivalents aux pertes et s'ajoutent à ceux qui étaient déjà produits avant l'action compensatoire (état de référence) » (Pioch, $2017: 40$ ).

2. Par traduction, nous entendons le processus de transposition, par des acteurs y ayant intérêt, des éléments d'un cadre cognitif d'une notion en principes normatifs, techniques et logiques ayant vocation à appréhender le réel pour l'évaluer et/ou agir sur ce dernier de manière pratique et mesurable (Lascoumes, 1994 ; Villalba, 2009). La traduction de la compensation désigne ainsi les mesures compensatoires telles qu'elles sont proposées par les cabinets de consultants.

3. L'effondrement des espèces et de leurs effectifs sur nos territoires (Mace et al., 2014) provoquent des irréversibilités, car l'accélération de la crise de biodiversité (Barnosky et al., 2012) ne permet pas à celui-ci de trouver les conditions de sa permanence.

4. «[... la mise en place de mesures de compensation peut occasionner une pluralité d'opérations écologiques que le droit ne saurait prévoir. Comme les obligations de remise en état, elles sont donc fixées au cas par cas, le plus souvent par des experts. Les bureaux d'études proposent une première mouture, laquelle sera ensuite discutée par les services administratifs, le public, et le cas échéant, par des comités scientifiques, avant d'être approuvée ou modifiée par l'autorité administrative compétente » (Lucas, 2015 : 37).

5. Élus (députés ou sénateurs) (2), hauts fonctionnaires travaillant au sein du ministère de l'Environnement ou du Commissariat général au développement durable (4), de consultants spécialistes de la compensation ou de l'évaluation environnementale (14) ainsi que de chercheurs universitaires (2) ou encore de représentants, salariés ou non, d'associations naturalistes (2). Ces acteurs ont été sélectionnés du fait de la récurrence de leur présence dans les débats (soit physiquement soit en étant cités), du fait de leurs positionnements professionnels (décisionnaire, rapporteur de loi, consultant) et de leur capacité à s'établir dans le champ commercial de la compensation (chiffre d'affaires du cabinet de conseil, présence sur le territoire, capacités techniques et financières...).

6. http://www.labex-cemeb.org/fr/actualites/colloque-eviter-reduire-compenser ; http://www.js.univ-nantes.fr/colloque-6-la-compensation-ecologique-en-

questions-1494446.kjsp ; https://www.afbiodiversite.fr/fr/actualites/eviter-reduirecompenser-seminaire-sur-la-phase-devitement.

7. Le colloque Éviter, Réduire, Compenser qui s'est déroulé du 30 au 31 mars 2017 (Montpellier, Agropolis International, Labex Cemeb), le séminaire sur la phase d'évitement de la séquence "Éviter, Réduire, Compenser » (ERC) organisé le 19 avril 2017 (Paris, La Défense, ministère de l'Environnement, de l'Énergie et de la Mer) et le colloque La compensation écologique en questions, organisé le 2 juin 2017 (Nantes, Centre des congrès, IFSTTAR, IUT de Nantes).

8. Entretien mené le 21 février 2017, Assemblée nationale, Paris.

9. Entretien mené le 21 février 2017, Assemblée nationale, Paris.

10. Christopher Hood (1995) identifie sept «variations» des politiques managériales reposant sur un principe commun de bonne gestion des deniers publics : la division du secteur public en unités à des fins de gestion, la mise en concurrence de ces unités et la mise en concurrence des organisations publiques et privées, l'intégration des techniques de management issues du privé, la réduction des coûts de fonctionnement, l'efficience dans les consommations, le raccourcissement des lignes hiérarchiques, l'intégration d'objectifs de performance contrôlables et la culture du résultat. 
11. La multiplicité de ces « unités de compte» des mesures compensatoires, impulsée par la nécessité de justifier le « pas de perte nette » est symptomatique de la difficulté à trouver une modalité d'expression unifiée de la restauration de la biodiversité. Comme l'explique cette chargée de mission, haut fonctionnaire, rencontrée en marge du Forum Biodiversité et économie, vers une agence française pour la biodiversité, qui s'est tenu les 24 et 25 novembre 2016 à Paris : "Il me semble qu'on ne peut pas tout d'un coup trouver la pierre philosophale, on n'a pas comme pour le carbone quelque chose d'uni qui est la tonne équivalent $\mathrm{CO}_{2}$, on n'a pas [pour la biodiversité] une unité physique homogène, donc la question est beaucoup moins simple. »

12. Entretien avec un consultant spécialisé en évaluation environnementale et effectuant à ce titre des évaluations de mesures compensatoires, principalement pour des aménageurs privés (tels que Eiffage ou Vinci), Paris, 21 mars 2017.

13. Soit directement (capital humain) soit indirectement, le capital technique étant issu, dans un premier temps, de la main de l'homme.

14. Ce stock étant alors nommé « capital naturel critique » (De Groot et al., 2003).

15. Que cette époque soit considérée dans le temps commun - le temps calendaire - ou qu'il s'agisse d'une époque calibrée par l'artiste - la période bleue chez Picasso par exemple.

16. Des économistes (Randall, 1991), philosophes (Maris, 2010), éthiciens (Callicott, 2010) proposent à ce titre d'identifier différentes valeurs du vivant telles que les valeurs esthétiques, récréatives, juridiques, etc.

17. Entretien avec un universitaire économiste spécialiste des questions de compensation, Lille, 26 janvier 2017.

18. Entretien réalisé avec un ingénieur, créant un outil de restauration écologique à des fins de commercialisation sur la base de l'outil bilan carbone, Paris, 21 février 2017.

19. Intervention d'une salariée travaillant pour une association de défense de l'environnement lors de l'atelier «L'évaluation socio-économique des projets d'aménagement : comment améliorer le dispositif pour qu'il prenne mieux en compte la biodiversité ?» lors du Forum Biodiversité et économie, vers une agence française pour la biodiversité, 25 novembre 2016, Hôtel de Roquelaure, Paris.

20. Hicham-Stefan Afeissa, "Entretien avec Holmes Rolston III", 10 juin 2008, disponible à l'adresse https://www.nonfiction.fr/article-1196entretien_avec_holmes_rolston_iii.htm, consulté le 28 septembre 2017.

21. Extrait d'une discussion lors d'un temps de repas organisé lors du colloque Éviter, Réduire, Compenser, Agropolis International, Montpellier, 30 mars 2017.

22. Nous ne souhaitons pas entrer ici dans le débat quant à la financiarisation du vivant mais bien montrer que toute substitution du capital naturel par du capital technique présuppose un coût, celui du remplacement généré par les éléments de compensation mis en œuvre lors de l'opération.

23. Il convient de noter que ce cabinet s'est concentré sur les mesures compensatoires, les mesures d'évitement et de réduction ayant consisté à établir le tracé le moins impactant sur la biodiversité pour un ouvrage représentant près de 107 kilomètres linéaires et estimé à 4,5 milliards d'euros.

24. La commission a entendu des bureaux d'études spécialisés, des opérateurs, experts naturalistes de conservatoires naturels, représentants du monde agricole, etc. ; certains 
membres de la commission se déplaçant même parfois sur place comme cela a été le cas sur un chantier de la ligne grande vitesse Tours-Bordeaux.

25. http://www.senat.fr/rap/r16-517-1/r16-517-11.html\#toc1.

26. Les propositions de la commission d'enquête s'organisent autour de neuf éléments : une meilleure connaissance de la biodiversité et une précision des phénomènes de fragmentation des milieux, une plus grande anticipation et une meilleure application de la séquence " ERC », des méthodologies à améliorer pour une mise en œuvre souple et efficace de la compensation, s'appuyer sur les collectivités territoriales pour une véritable planification de la compensation, un renforcement des expertises publique et privée, une meilleure intégration du monde agricole à la compensation, la création du consensus autour de l'expertise scientifique, une transparence des coûts de la séquence «ERC », une amélioration du suivi dans le temps des mesures de compensation (Dantec, 2017).

\section{RÉSUMÉS}

La compensation écologique telle que traduite par les cabinets de conseil depuis la loi sur la biodiversité de 2016 s'adapte aux logiques de performance du New Public Management. Les mesures compensatoires qui en découlent reposent sur les principes de quantification sélective et de substituabilité de la biodiversité, qui contribuent à réduire la complexité des interdépendances entre milieux sociaux et milieux naturels. Cela contribue à valoriser la valeur économique de la biodiversité au détriment de sa valeur systémique. Nous souhaitons montrer le décalage entre, d'une part, la création de l'instrument " compensation » destinée à lutter contre l'érosion de la biodiversité et, d'autre part, les conséquences de sa mise en œuvre sur la reconnaissance des irréversibilités écologiques.

Since the biodiversity law in 2016, biological offsetting, which is traduced by consulting firms, is an adaptation of performance logic induced by New Public Management. Biodiversity offsets are determined by consulting firms. It is based on quantification and substitutability of biodiversity, which contributes to reduce complexity of interdependencies between natural environment and social environment in order to respond to NPM goals of efficiency and results. It aims at promote economical value of biodiversity at the expense of ecosystemic value. In a context of biodiversity crisis, we should emphasize the shifting between biodiversity offsetting tool (in order to contain biodiversity crisis) and their irreversible ecological consequences.

\section{INDEX}

Mots-clés : compensation, New Public Management, quantification, substituabilité, irréversibilités

Keywords : offsetting, New Public Management, quantification, substituability, irreversible consequences 


\section{AUTEURS}

\section{RÉMY PETITIMBERT}

Rémy Petitimbert est doctorant et ATER en science politique à l'université de Lille. Sa thèse porte sur l'instrumentation des politiques nationales de biodiversité en France à partir de l'exemple de la compensation écologique. CERAPS/CNRS (UMR 8026).

\section{CLÉMENCE GUIMONT}

Clémence Guimont est doctorante à l'université de Lille et ATER à l'Institut des administrations et des entreprises de l'université de Lorraine. Sa thèse porte sur la sociologie de l'action publique territoriale de biodiversité. CERAPS/CNRS (UMR 8026). 